\title{
CONSTRUCTION ACTIVITY MODELLING FOR AUTOMATION
}

\author{
Sotiris A. Pagdadis \\ Ph.D. Candidate, Dept. Civil Engineering, The University of Texas at Austin \\ James T. O'Connor \\ Assistant Professor, Dept. Civil Engineering, The University of Texas at Austin
}

\begin{abstract}
Modelling of global work environments requires the implementation of techniques capable of explicitly defining and capturing interrelationships amongst the individual components that comprise the system. A construction environment creates particular modelling challenges that are a result of its loosely defined working infrastructure. In a detailed effort to develop a methodology for evaluating the automation potential of construction activities, and the consequent assessment of appropriate technology strategies, a model has been developed to depict human-machine interactions for construction work. The model structure follows a systems analysis approach, as would be common in industrial automation, with deliberate considerations for the peculiarities of the construction world.
\end{abstract}

\subsection{INTRODUCTION}

The field of construction automation has recently been receiving considerable attention both from industry and academia all over the world. [TUCKER88a] Automation and robotics applications are not commonplace on the construction job-site, though there is considerable agreement that the needs for their implementation are high, and once implemented will facilitate the construction environments of the future. There is further consensus that methodological evaluation data of such needs must be gathered. [KANGARI88] [PAGDADIS88] [SKIBNIEWSKI88] [TUCKER88b]

The construction environment, hostile as it may seem towards the implementation of fully automated processes, offers a plethora of areas where technology assessments from alternative fields can be implemented to accommodate component technology needs in construction. Implementation of robotic technology will not occur unless fundamental procedural changes are introduced. Constructability techniques promoting "design for construction" should be further applied to "design for automation" before a less hostile construction work place can be visualized. Extreme cases, such as the "Three Mile Island" spill, have provoked some progress, though old traditions are still controlling growth. With time, milestone technology improvements will be made, where the physical manual contribution will decrease, and the automated contribution will grow.

To gather evaluation data, the environment must be modelled at a level of detail which provides meaningful measurement data, while at the same time avoiding meaningless abstractions from the real world. Since the ultimate goal is to assess technology strategies at a component level, the modelling technique must be capable of depicting elementary, yet cognitive, levels of representation. A systems approach has been chosen to develop the 
model structure for construction. The modelling technique is described as a Construction Activity Model (CAM), to characterize the specific representational features for construction.

This paper focuses on the needs for construction activity modelling, the specific representation of the CAM, and its implementation.

\subsection{THE SYSTEMS APPROACH}

A system is a set of objects together with relationships between the objects and between their attributes. [DEGREENE70]

Systems analysis is the basic technique used to define equipment, personnel, facilities, and procedural data required to meet the system objectives. The first step in the approach is to recognize that the problem can be solved using a systems analysis approach. It requires a definition of the system using its hierarchical representation, functions, interactions and goals. It further requires the definition of measurement criteria, and consequent knowledge acquisition and data analysis of operational systems.

A human-machine system is a particular type of system, defined as an operating combination of people with equipment. It is broken down into its components, depicting all functional interactions, information and decisions.

\subsection{The Modelling Paradigm}

A model is a specific representation of a system. The representation that is of concern here, is that of the human-machine interactions in the construction environment. The representation may be symbolic, physical, mathematical, or diagrammatic. The structure of the model may be either analytical, or conceptual. Analytical models are computational formulations that define the human-machine interaction. Conceptual models are interpretive formulations that conceptualize the human-machine interaction. The purpose of the model may be descriptive, or normative, depending upon the particular goal. Descriptive models attempt to explain the operator's cognitive behavior. Normative models tend to specify the operator's control in either a prescriptive or operational manner.

Modelling a human-machine system, requires a specification of the human-machine interaction. A device-directed model specifies a dependency on particular equipment. Generalization of equipment needs and uses, requires a focus of the model on the task to be accomplished. Such a model is task directed. Task-directed models attempt to characterize the manner in which the human interacts with the system. The Construction Activity Model encompasses this approach.

\subsection{CONSTRUCTION ACTIVITY MODEL DEVELOPMENT}

The purpose of the Construction Activity Model is to describe the operator's cognitive behavior within an ill-structured environment. Structuring of the working environment is difficult to achieve. The activities are often time disjointed and highly diverse. The model of the system is operator-oriented. It represents the inputs and outputs of the working environment in terms of the actions and decisions of the operator, and the information resources necessary for processing. The model depicts the human-machine interaction using a behavioral classification which is independent of specific technology. A concerted effort is made to generalize device specificity. 


\subsection{The Construction Taxonomy}

A hierarchical representation organizes the system into its component parts. Figure 1 portrays the representation of the construction environment in a similar manner to that taken to describe the work breakdown structure.

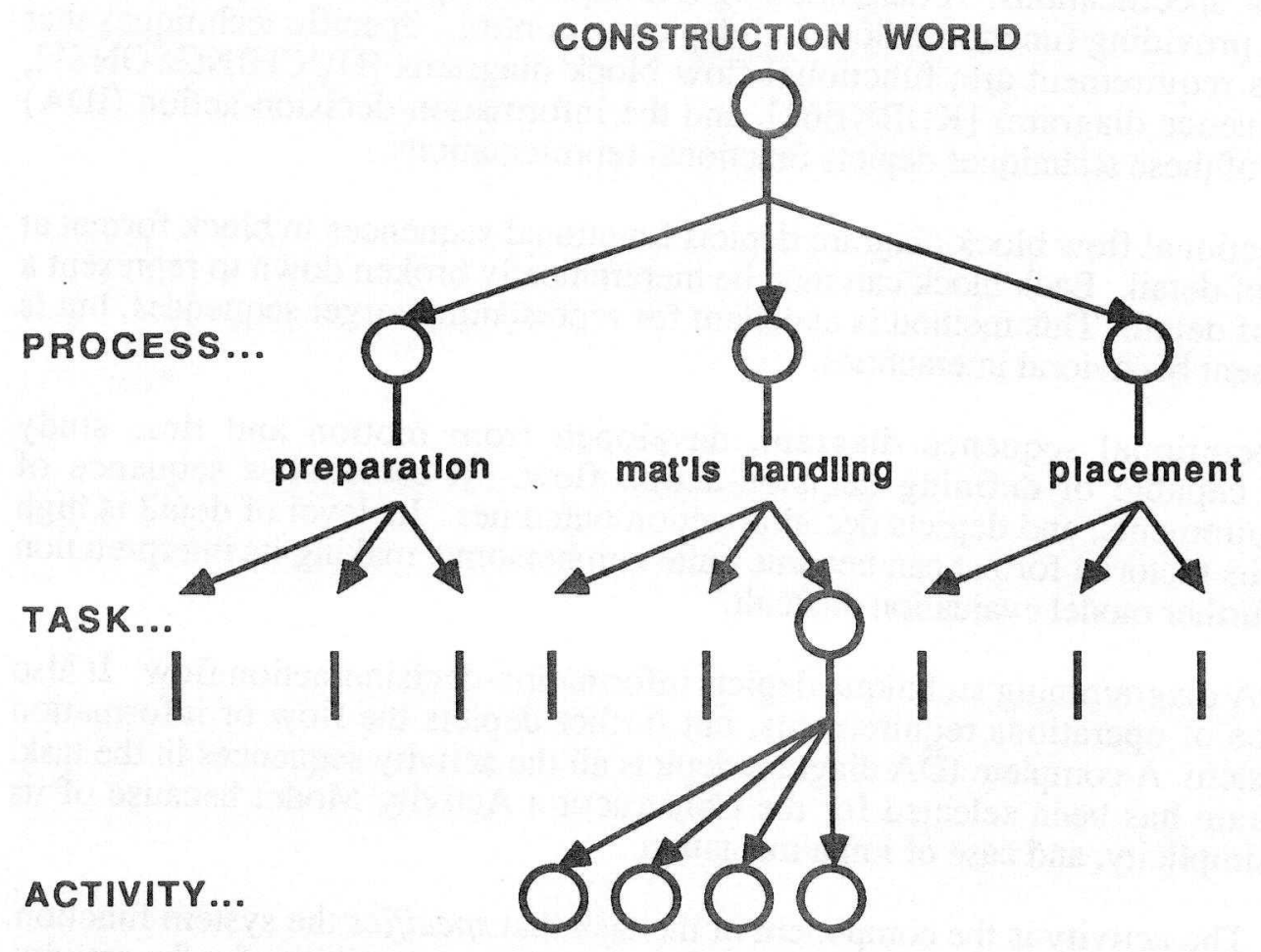

Figure 1: Construction Classification Hierarchy

Construction World: The construction world depicts the head of the taxonomy. It is described as the parent environment and provides specificity to the model. Construction world examples include; industrial piping, structural, mechanical, electrical, etc.

Process: The process categorizes the job operation to be conducted. The construction environment is categorized into three processes:

- materials preparation
- $\quad$ materials handling
materials placement

Each of these categories is more specifically defined by the dependency on the construction environment. For example, in heavy industrial piping construction, these processes represent; fabrication, transport and staging, and erection, respectively.

\subsubsection{Task Analysis}

Having categorized the job operations as processes, the next step is to sequentially document the tasks that fully carry out functional representations. A task is a group of activities that occur in temporal proximity, and often sequentially. The task level 
analysis allows systematic study of the behavioral requirements of the tasks. This analysis should be viewed as a means for system understanding and data gathering, and not as an end in itself.

Task: The task description provides a means for defining human-machine needs, interactions, and goals. Other equipment requirements may also be identified, such as tools, check lists, specifications. A diagramming technique is employed to capture the task descriptions by providing functional flow and depicting control. Specific techniques that accomplish this requirement are; functional flow block diagrams [HUCHINGSON81], operational sequence diagrams [KURKE61], and the information-decision-action (IDA) diagram. Each of these techniques depicts functional representations.

The functional flow block diagram depicts functional sequences in block format at specific levels of detail. Each block can then be hierarchically broken down to represent a separate level of detail. This method is excellent for representing target sequences, but is unable to represent behavioral interactions.

The operational sequence diagram, developed from motion and time study techniques, is capable of defining decision-action flow. It establishes sequence of operations requirements, and depicts decision-action outcomes. Its level of detail is high and as a result its pictorial format can become quite cumbersome, making its interpretation complex, and further model evaluation difficult.

The IDA diagramming technique depicts information-decision-action flow. It also shows sequence of operations requirements, but further depicts the flow of information through the system. A complete IDA diagram depicts all the activity sequences in the task. The IDA diagram has been selected for the Construction Activity Model because of its functionality, simplicity, and ease of implementation.

Activity : The activity is the component of the task that specifies the system function. The functionality does not necessarily imply human operation responsibility for the activity, neither does it necessarily mention specific equipment use. The function statement implies a goal. This structure is best represented in the IDA diagram.

\subsection{IDA Functional Form}

Each activity in the IDA diagram is portrayed by information feedback, a decision cue, and an action. The function statement is identified in the action. It consists of a verb/noun combination and descriptors, where the verb portrays the stimulus, response, or organism behavior. The action is predominantly characterized as a physical response. The response is instigated by a stimulus, which in the case of the IDA diagram can be portrayed by another action, a decision cue, or an activity flag. The organism is also portrayed in the action, and defines the processing of information depicted in the activity. The response is always depicted in the action, the stimulus is depicted in the decisionaction, while the organism is depicted in the information-action.

The strongest feature of the IDA diagram is its ability to portray the activity sequences vertically, through the action, while at the same time depicting the information flow independently for each activity. Figure 2 portrays a typical sequence of activities.

The symbolism of the IDA diagram is elementary. The sequence is indicated by Arabic numerals, which are also later used as identifiers for activity evaluation. [PAGDADIS88] Each sequence contains a decision-action. Many activities also 
incorporate information flow requirements. Flags indicate decision controls sequence flow. The actions contain a verb/noun combination and descriptors;

eg.prepare(v)__closures $(n) \quad$ for transport $(d)$

The verb depicts the functional behavior of the action, i.e. as a stimulus-organism-response characteristic. The noun portrays the object, and the descriptor portrays its context.

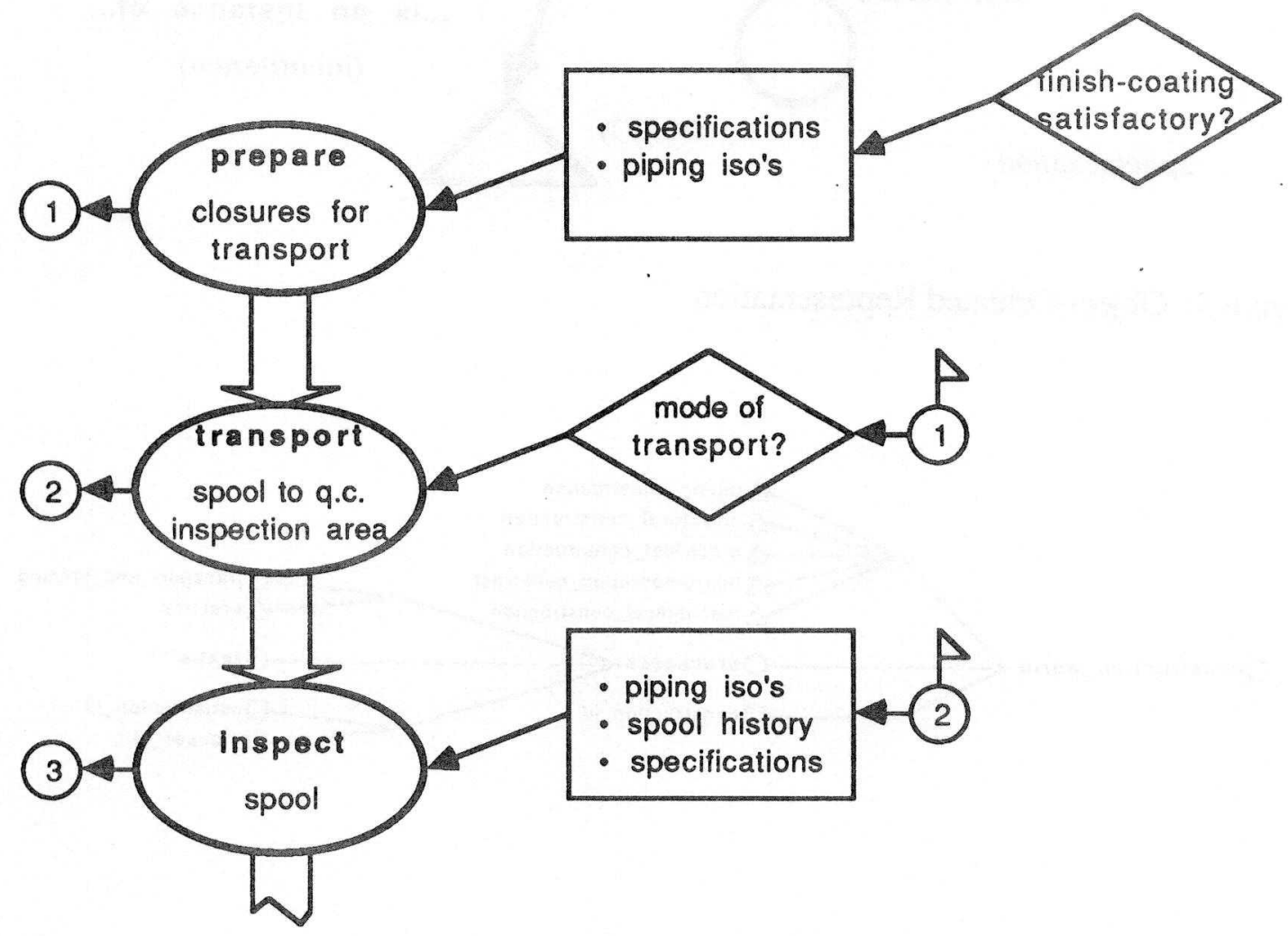

Figure 2: IDA Sequence

\subsection{CAM Representation}

Components of the system hierarchy are objects. The object is a structured symbolic description with defined characteristics. A class is a collection of objects sharing common properties. It is a generalization of the object. The object, therefore, is a specialization of the class. Figure 3 portrays this categorization.

The object-oriented representation is the most appropriate approach to computationally represent the CAM. Each level of the hierarchical taxonomy is identified as a class with sub-classes, objects and properties. The objects are instances of the class, and as a result can inherit its properties. Inheritance is a very powerful feature of the object-oriented representation. An additionally powerful feature of this representation is the ability objects have to inherit functions. These functions are called methods. Figure 4 shows examples of this representation that have been applied to piping construction. 


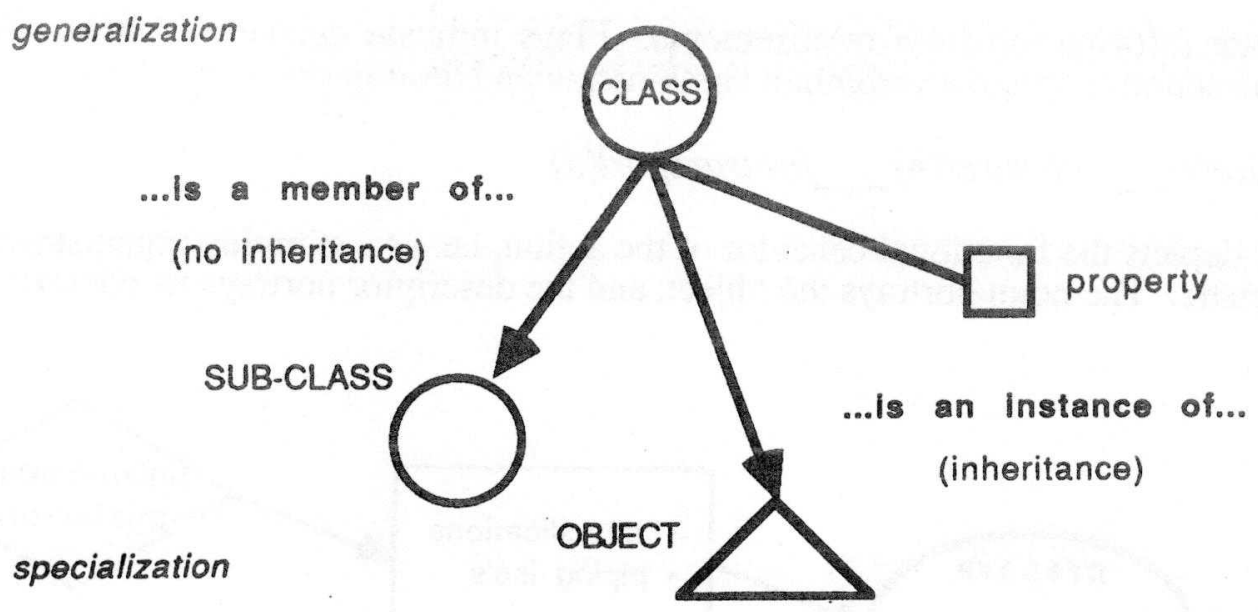

Figure 3: Object-Oriented Representation
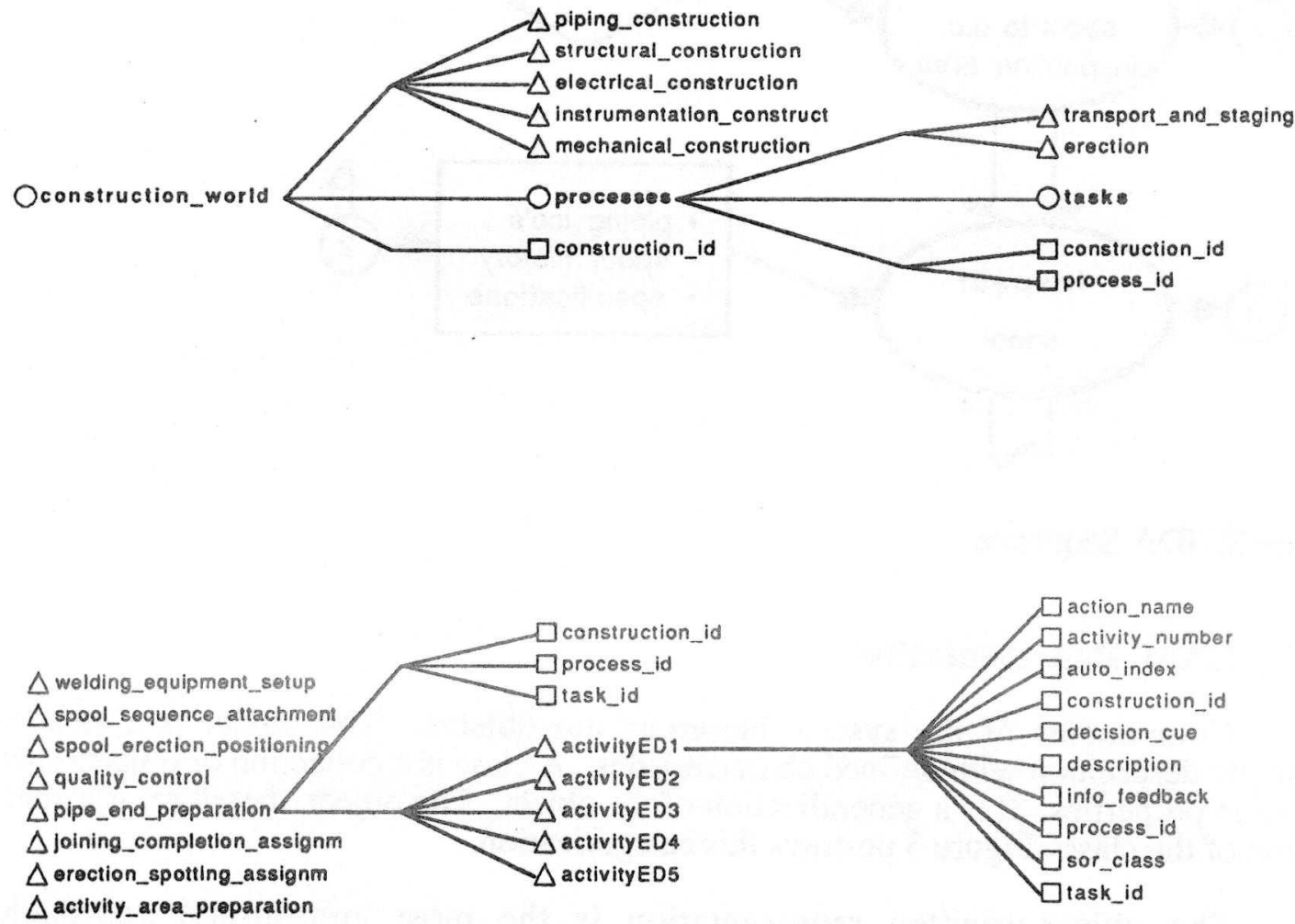

Figure 4: Object-Oriented Representations for Piping Construction 
The IDA diagram is computationally represented as a form. The computer image space is sensitive to the location of the object sequences in the form. Each task is depicted separately. The activities in the sequence have stored properties that can be retrieved, modified, or queried.

\subsection{CAM IMPLEMENTATION}

The Construction Activity Model has provided a methodology for modelling construction sequence activities, defining functional property requirements, interrelationships, and representing information flow. Its computational format is used as a launch model for several applications.

\subsection{Technology Assessment Strategy for Construction (TASC)}

In an effort to assess component technology needs for construction activities, a multi-attribute decision model has been designed as a tool for knowledge acquisition. The model focuses on concerns in productivity, safety, quality, and super-human handling. [PAGDADIS88] The IDA diagrams are used as a basis for evaluating human-machine needs for each construction activity. Collected data is analyzed, validated, and computationally stored as activity properties.

Advanced component technology software and hardware specifications are documented in a knowledge base. They address the individual attributes in the decision model. An artificial intelligence pattern matching strategy is then conducted to assess technology strategies for each activity, based on the property needs.

\subsection{Site Operations Control System (SOCS)}

The most powerful feature of the Construction Activity Model is its ability to capture hierarchical interrelationships, and document information flow through the IDA diagram. The computational form of the IDA diagram stores its data in a knowledge base. This data can be queried to provide important site level management information, including, detailed activity sequencing, material allocation, manpower and equipment utilization, control of change operations, and quality control data, amongst others. These features are essential in the evolution of the automation process.

\subsection{CONCLUSIONS}

The Construction Activity Model provides a framework for representing construction work environments. It has provided a basis for portraying the humanmachine element. The robustness of the Information-Decision-Action diagram and its representation, has enabled the detailed modelling of construction activity sequences. Its implementation is diverse. It has been used as a visual communication tool for evaluating the automation potential of construction activities, and as a visual computational tool for decision support management of technology assessments. This work has been applied to heavy industrial piping construction.

\section{Acknowledgements} 8615105 .

This work is funded by the National Science Foundation under grant number DMC- 


\section{REFERENCES}

\section{[DEGREENE70]}

DeGreene, K.B., Systems Psychology, McGraw Hill, New York, 1970, p. 6.

\section{[HUCHINGSON81]}

Huchingson, R.D., New Horizons for Human Factors in Design, McGraw-Hill, New York, 1981, p. 24.

\section{[KANGARI88]}

Kangari, R., Halpin, D.W., "Automation and Robotics in Construction: A Feasibility

Study," The 5th International Symposium on Robotics in Construction, June 6-8, 1988, pp. 161-171.

\section{[KURKE61]}

Kurke, M.I., "Operational Sequence Diagrams in System Design," Human Factors, 3(1), 1961, pp.66-73.

\section{[PAGDADIS88]}

Pagdadis, S.A., "A Methodology for Building Information Support in Construction Automation," 1988 Seminar/ Symposium of the Project Management Institute, San Francisco, California, September 17-21, 1988.

\section{[SKIBNIEWSKI88]}

Skibniewski, M.J., "Framework for Decision-Making on Implementing Robotics in Construction," Journal of Computing in Civil Engineering, 2(2), ASCE, April 1988.

\section{[TUCKER88a]}

Tucker, R.L., "Perfection of the Buggy Whip," Journal of Construction Engineering and Management, 114(2), ASCE, June 1988, pp. 158-171.

\section{[TUCKER88b]}

Tucker, R.L., "High Payoff areas for Automation Applications," The 5th International

Symposium on Robotics in Construction, June 6-8, 1988, Tokyo, Japan, pp. 9-16. 